\title{
La politique éolienne au Québec : un mégaprojet?
}

\author{
Yann Fournis et Marie-José Fortin ${ }^{1}$
}

\section{INTRODUCTION}

\section{L'osmose entre territoires et grands projets semble appartenir au passé, parce que les performances technologiques croissantes réduisent sans cesse les retombées économiques notamment en termes de ratio investissement/emploi, alors que des considérations sociales et environnementales suscitent des protestations.}

Longtemps considérés comme la voie royale du développement des territoires au Canada, les grands projets tiennent de l'évidence pour nombre de régions « ressources » ou «périphériques », historiquement structurées par l'omniprésence de grandes entreprises. Toutefois, cette évidence devra sans doute faire ses preuves, tant les rapports entre grands projets et territoires semblent se transformer.

D'un côté, le contexte de mondialisation et de mobilité des capitaux tend plutôt à renforcer l'exploitation des ressources et la compétition autour d'elles (entre grandes entreprises, mais aussi entre territoires, qui se veulent toujours plus attractifs à coup de politiques ad hoc). D'un autre côté, l'osmose entre territoires et grands projets semble appartenir au passé, parce que les performances technologiques croissantes réduisent sans cesse les retombées économiques notamment en termes de ratio investissement/emploi ${ }^{2}$, alors que des considérations sociales et environnementales suscitent des protestations. Il y a là un paradoxe pour les territoires: les avantages historiques des grands projets s'amenuisent sans cesse alors que leurs coûts restent singulièrement élevés...
Pour dépasser ce paradoxe, l'on examinera ses termes de manière dynamique, en considérant que les territoires et les grands projets sont sans doute en train d'évoluer simultanément, dans le cadre d'une vaste mutation de l'économie politique des ressources naturelles. Selon certains travaux inspirés par la théorie des principales ressources, il se produirait une transition générale du modèle canadien de développement, qui deviendrait de plus en plus post-staples, mais selon des formes, rythmes et amplitudes différents selon les ressources, secteurs et territoires. Cette tendance, que nous avons pu observer récemment en comparant les secteurs forestier, de la production porcine et de l'énergie éolien$\mathrm{ne}^{3}$, justifie que l'on observe ensemble les transformations des mégaprojets et des régions ressources québécoises pour saisir, dans des cas concrets, la recomposition de leurs rapports.

À propos du mégaprojet que représente la politique éolienne du Québec, la démonstration sera menée en quatre points :

- quelques repères sur la théorie des principales ressources rappelleront que la production éolienne apparaît dans une période de recomposition de l'exploitation des ressources (en l'occurrence hydroélectriques);

- une description de la politique éolienne indiquera que, en dépit de sa complexité, elle correspond largement à ce que la littérature considère comme un «mégaprojet »;

- l'examen de la gouvernance du projet éolien permettra de comprendre si ses processus et mécanismes participent de logiques nouvelles d'exploitation des ressources;

- l'étude de la place ambiguë que les acteurs territoriaux y occupent. 


\section{UNE HYPOTHÈSE : LA TRANSITION DE L'ÉCONOMIE POLITIQUE DES RESSOURCES}

Le développement territorial au Canada a longtemps été lié à la commercialisation et à l'extraction des ressources naturelles et aux activités connexes (foresterie, mines, pêche et agriculture). La théorie des ressources premières d'Harold Innis analyse dans la longue durée (depuis le $\mathrm{XVI}^{\mathrm{e}}$ siècle) comment la formation des marchés du Nouveau Monde s'est coulée dans les circuits internationaux du capitalisme marchand. Il en a résulté une dépendance aux forces exogènes ce qui, en dépit de facteurs favorables (maind'œuvre formée, épargne domestique, etc.), a défavorisé la constitution d'une économie intégrée, propre et cohérente (marché domestique, industries fortes, etc. $)^{4}$. T. Barnes résume la tendance : les secteurs basés sur l'exportation des ressources primaires suscitent un blocage du processus de diversification économique (pour des raisons diverses: culture d'exportation, domination des grandes multinationales, structures industrielles tronquées) et créent un «piège des principales ressources ». L'industrialisation du Canada a donc été historiquement bloquée par son insertion dans des types de commerces instables, peu avantageux, directement soumis à la volatilité des marchés internationaux et à la domination de grandes entreprises $^{5}$. Cependant, depuis la fin $d u$ $\mathrm{XX}^{\mathrm{e}}$ siècle, les signes d'une crise s'accumulent.

Selon certains, les provinces canadiennes seraient entrées dans une nouvelle phase de développement, dite «post-staples », parce que la maturation des régimes (fordisme, mécanisation) a créé de nouvelles pressions (épuisement de et pression des entreprises sur la ressource, essor de résistances sociales, intensification capitalistique et technologique de l'exploitation, diversification économique) $)^{6}$. L'impact de ces variables, nou- velles et multiples (technologiques, économiques, sociales, politiques, etc.) remettrait en cause la relative solidité et simplicité du modèle traditionnel de développement économique. Mais ceci signifie moins la disparition du rôle des ressources naturelles que l'émergence d'une économie plus complexe et nuancée, où de nouvelles tendances économiques (essor de l'économie du savoir, des services et industries de la connaissance, etc.) se combinent et pénètrent les logiques de secteurs traditionnellement associés aux ressources premières (agriculture, foresterie, mines, énergie). Bref, les provinces canadiennes relèvent encore d'une économie dépendante des ressources, mais de manière de moins en moins exclusive et selon des logiques de plus en plus hybrides. Le développement de la récente filière éolienne s'inscrit bien dans cette tendance, illustrant la transformation du régime hydroélectrique dans le sens d'une diversification des ressources pertinentes (dont le vent), des modes de gouvernance (avec l'irruption du privé face au couple traditionnel État-Hydro-Québec) et des ancrages territoriaux des projets (sous la forme de parcs multiples).

\section{L'osmose entre territoires et grands projets semble appartenir au passé, parce que les performances technologiques croissantes réduisent sans cesse les retombées \\ économiques notamment en termes de ratio investissement/emploi, alors que des considérations sociales et environnementales suscitent des protestations.}

\section{LA POLITIQUE ÉOLIENNE AU QUÉBEC COMME MÉGAPROJET}

\section{La politique éolienne du Québec est donc un mégaprojet d'exploitation d'une ressource nouvelle (le vent), qui vise un développement industriel en alliant différents volets (industriel, énergétique et territorial).}

Introduite au sein du régime hydroélectrique québécois lors de sa redéfinition de la fin des années 1990, l'énergie éolienne apparaît comme une «ressource complémentaire» dans un portefeuille énergétique dominé par l'hydroélectricité ${ }^{7}$. À ce titre, la production éolienne relève d'un sous-secteur du régime hydroélectrique, dont elle prolonge les grandes orientations, et en particulier la volonté de conjuguer production énergétique, production industrielle et développement territorial. Ainsi, le volet principal de la 
politique sera industriel: un programme public doit générer une industrie produisant des retombées économiques - et d'abord dans une zone couvrant la MRC de Matane et la péninsule gaspésienne. Mais la politique éolienne aura aussi un volet énergétique, puisque l'implantation de parcs de grande taille sur une courte période doit stimuler un marché pour attirer au Québec les grandes entreprises étrangères possédant la technologie. C'est en ce sens que la politique éolienne, dans son ensemble, est un seul mégaprojet, mais diffracté en plusieurs sites.

Mais il ne faut pas s'y tromper: les projets énergétiques sont d'ampleur de plus en plus imposante (y compris les parcs éoliens ${ }^{8}$ ) et privilégient désormais une forte concentration spatiale. Initialement, certains observaient que la « nouvelle électricité » (solaire et éolienne) prenait une forme d'exploitation et une empreinte spatiale plus diffuses que les projets liés aux ressources plus traditionnelles (le «nouveau » pétrole : gaz de schiste et sables bitumineux $)^{9}$. Mais cette différence s'estompe et certains projets se rapprochent de la définition typique du mégaprojet ${ }^{10}$ : le parc du Lac-Alfred près d'Amqui (MRC de la Matapédia et de la Mitis) ouvert en septembre 2013 représente 150 éoliennes, 300 MW de puissance installée, 700 millions de dollars d'investissement et l'association de trois multinationales (l'albertaine Enbridge, la française EDF Énergies Nouvelles et l'allemande Repower ${ }^{11}$ ).

Globalement, la politique éolienne du Québec est donc un mégaprojet d'exploitation d'une ressource nouvelle (le vent), qui vise un développement industriel en alliant différents volets (industriel, énergétique et territorial), et se traduit dans l'espace par une empreinte relativement diffuse (dans une vingtaine de sites, en service ou en construction en 2013), qui a représenté un investissement total de 5 milliards depuis ses premiers pas $^{12}$. Si l'on admet que la politique éolienne est une sorte de mégaprojet, d'inspiration publique, mais largement mis en œuvre par le privé, il reste à saisir dans ses finesses et contradictions ce que la gouvernance de ce mégaprojet nous apprend sur la transformation des rôles des uns et des autres dans l'exploitation des ressources naturelles.

\section{LA GOUVERNANCE DU MÉGAPROJET ÉOLIEN}

\section{Le mégaprojet éolien évoque l'image d'un État qui se veut entrepreneur : il vise à l'industrialisation d'une ressource nouvelle dans le giron du secteur hydroélectrique, selon des objectifs territoriaux ambitieux et courageux (relancer une « région- \\ ressource ») et des paramètres d'une grande finesse (clauses de contenu industriel territorial), incarnés par un instrument flexible (l'appel d'offres) pour enrôler l'expertise des grandes entreprises internationales.}

Une première analyse de la gouvernance éolienne, centrée sur l'échelle nationale et une perspective top-down, ne peut manquer de constater combien le volet industriel de la politique est innovant bien plus que le volet énergétique - et en particulier dans son rapport aux territoires. Le mégaprojet éolien évoque l'image d'un État qui se veut entrepreneur: il vise à l'industrialisation d'une ressource nouvelle dans le giron du secteur hydroélectrique, selon des objectifs territoriaux ambitieux et courageux (relancer une «régionressource ») et des paramètres d'une grande finesse (clauses de contenu industriel territorial), incarnés par un instrument flexible (l'appel d'offres) pour enrôler l'expertise des grandes entreprises internationales. Toutefois, le volet énergétique du mégaprojet est plus décevant, illustration des limites classiques d'un État régulateur conforme au style traditionnel des politiques canadiennes liées aux ressources. En témoigne le système d'appel d'offres, qui confie aux promoteurs privés l'insertion locale des projets tout en assurant la protection technique et financière $\mathrm{du}$ régime hydroélectrique (par la priorité accordée aux critères de la connexion au réseau et du tarif d'achat aux producteurs).

Cette logique a-territoriale, où l'État pilote les territoires par les instruments et la technique, se nuancera progressivement, avec l'introduction de critères territoriaux quant à l'implantation des parcs (pour $25 \%$ de la pondération... contre $30 \%$ pour le coût de l'électricité), qui donnera (un peu) plus de poids aux acteurs territoriaux. De manière 
générale, il est possible de qualifier la gouvernance du mégaprojet éolien, en s'inspirant librement de travaux sur les politiques publiques liées aux ressources $^{13}$. La politique éolienne est une configuration de trois cercles d'influence inégale (Tableau 1). Au centre, un milieu décisionnel resserré rassemble un réseau néo-corporatiste de politique publique (comptant les seuls acteurs majeurs, publics et privés), entouré par la nébuleuse floue et élargie d'une commu-nauté pluraliste de politique publique (aux intérêts et représentations plus diversifiées) et un large halo, sans cohérence réelle, des prétendants à la participation ou à l'influence dans les politiques éoliennes. Puisque ces derniers sont essentiellement locaux, tournons-nous pour finir vers les acteurs territoriaux.

Tableau 1 - Configuration du sous-secteur éolien

\begin{tabular}{|c|c|c|c|}
\hline Échelle & Mécanisme & Action publique & $\begin{array}{l}\text { Secteur privé et } \\
\text { tiers secteur }\end{array}$ \\
\hline Nationale & $\begin{array}{l}\text { Conception de la } \\
\text { politique }\end{array}$ & $\begin{array}{l}\text { Gouvernement, } \\
\text { Régie de l'Énergie, } \\
\text { MRN, Hydro-Québec }\end{array}$ & $\begin{array}{l}\text { Multinationales } \\
\text { Entreprises locales }\end{array}$ \\
\hline Transversale & $\begin{array}{l}\text { Mise en œuvre de la } \\
\text { politique }\end{array}$ & $\begin{array}{l}\text { MDEIE, MDDEFP } \\
\text { (BAPE), MAMROT } \\
\text { CPTAQ }\end{array}$ & $\begin{array}{l}\text { Promoteurs } \\
\text { Associations de } \\
\text { producteurs et } \\
\text { d'entreprises } \\
\text { Réseaux d'expertise }\end{array}$ \\
\hline Locale & $\begin{array}{l}\text { Participation à la } \\
\text { politique }\end{array}$ & $\begin{array}{l}\text { Acteurs publics } \\
\text { locaux (CRE, MRC, } \\
\text { municipalité) } \\
\text { Producteurs } \\
\text { communautaires }\end{array}$ & $\begin{array}{l}\text { Producteurs } \\
\text { communautaires } \\
\text { Comités de citoyens } \\
\text { Propriétaires fonciers }\end{array}$ \\
\hline
\end{tabular}

Source : Fournis, Fortin, Prémont et al., 2013

\section{LA PLACE AMBIVALENTE DES TERRITOIRES DANS LA GOUVERNANCE DU MÉGAPROJET}

Le volet industriel de la politique éolienne est rapidement l'objet d'une mobilisation locale en Gaspésie, assez efficace, qui réplique la concertation nationale et démontre que la politique éolienne y trouve des relais solides (avec un embryon de politique énergétique régionale).

Le mégaprojet éolien peut aussi être examiné à l'échelle locale, en fonction des scènes territoriales de sa mise en œuvre. Ici, se révèle une configuration d'acteurs propre, très différente du niveau national, qui participe à une retraduction et une opérationnalisation de la politique publique au moment de sa mise en œuvre sur le terrain (la «production» de la politique). Cette scène, souvent négligée dans l'analyse, est importante parce que l'emprise des « grands » acteurs nationaux n'a pas la même portée sur la scène de mise en œuvre, où interviennent à la fois des instances nationales et des acteurs locaux. Ainsi, le volet industriel de la politique éolienne est rapidement l'objet d'une mobilisation locale en Gaspésie, assez efficace, qui réplique la concertation nationale et démontre que la politique éolienne y trouve 
des relais solides (avec un embryon de politique énergétique régionale). La réception locale $\mathrm{du}$ volet énergétique du mégaprojet est cependant très différente: dans des communautés locales, on retrouve des positions ambivalentes quant à l'implantation des parcs éoliens.

Certains territoires connaissent des résistances aux premiers développements éoliens, au mieux maladroits, et de vives controverses sociotechniques comme dans le Bas-Saint-Laurent (certains iront jusqu'au référendum local). D'autres territoires, à la même époque, se mobilisent pour partager la ressource : en Gaspésie, des élus locaux s'organisent au niveau de la région pour lancer des projets avec le privé (les «projets greffons») et suggèrent un modèle de partenariat inégalitaire qui se diffuse actuellement (dans la région voisine du Bas-Saint-Laurent). En tout état de cause, il n'apparait plus vraiment possible de penser l'installation d'équipements électriques sans prévoir la participation des acteurs locaux ou des retombées économiques substantielles pour les territoires.

\section{CONCLUSION : UN MODÈLE EN TRANSITION REMODELANT LES RAPPORTS CENTRE- PÉRIPHÉRIE}

Le modèle de développement lié à

l'exploitation des ressources misant sur les grands projets apparaît encore comme incontournable sur bien des territoires.

Le modèle de développement lié à l'exploitation des ressources misant sur les grands projets apparaît encore comme incontournable sur bien des territoires - comme en font preuve les nombreuses annonces qui tiennent souvent lieu de politique de développement économique. Néanmoins, ce modèle connait désormais des tensions parce que l'osmose qu'il présupposait entre grande entreprise et territoire est déstabilisée par divers facteurs contradictoires (technologies remplaçant la main-d'œuvre et diminuant les retombées directes, contestations croissantes des impacts sociaux et environnementaux, etc.). Au-delà des projets individuels, ces tensions désignent sans doute une évolution plus générale de l'économie politique canadienne: l'organisation de l'exploitation des ressources naturelles devra sans doute se renouveler pour perdurer. Telle pourrait être la leçon du mégaprojet éolien. Sa face éclairée offre le tableau d'un projet industriel audacieux, mu par une véritable ambition politique, inscrit dans une astucieuse diversification du régime hydroélectrique traditionnel et qui trouve un fort écho dans une région-ressource qu'il se propose de relancer...

Mais sa face cachée révèle aussi l'empreinte du style traditionnel des politiques de ressources sur ce mégaprojet, avec un volet énergétique défini quasi exclusivement par les élites du centre, en fonction de paramètres techniques a-territoriaux, qui n'ont pas vraiment pensé la phase de mise en œuvre (confiée au privé) et ont souvent récolté la contestation. Avec l'intégration croissante d'innovations nées à la périphérie, un nouvel équilibre entre acteurs nationaux et territoriaux semble se dégager actuellement, illustrant une insertion plus fructueuse du mégaprojet dans les milieux. Il nous semble que cette dernière évolution illustre assez bien non seulement les ambigüités de la transition économique actuelle, mais aussi sa dimension fondamentalement politique.

Revenons, pour finir, sur le sens délicat de la transformation $\mathrm{du}$ mode de développement canadien. D'une part, certains traits semblent renvoyer à une rupture du mode de développement, dans une nouvelle direction où les ressources naturelles occuperaient une place marginale dans l'économie, dominée par les dynamiques d'innovation et de création à base de connaissance, surreprésentées dans les zones centrales métropolitaines. Selon cette tendance, la sensibilité écologique, puissante dans les zones centrales, exclut une exploitation intensive des ressources au prix de l'environnement. Mais, d'autre part, d'autres évolutions semblent bien plutôt prendre racine dans une adaptation incrémentale du mode traditionnel de développement, où les ressources continuent à caractériser des pans entiers de l'économie canadienne, mais en fonction de logiques exacerbées (précarité des ressources, intensification technologique et financière, production diversifiée et flexible, etc.). 
Selon cette deuxième tendance, les espacesressources périphériques ont encore de beaux jours devant eux - mais à condition de se plier à une exploitation sans vergogne de leurs ressources. Ceci pose une question dramatique pour les territoires : comment pérenniser les communautés dans de telles conditions?

Pour interpréter cette évolution ambivalente, certains suggèrent que la transition du mode de développement reste ouverte ou hybride, parce que l'adaptation incrémentale des parties du système ne converge pas dans un paradigme de remplacement, cohérent et légitime. En conséquence, la gestion collective de cette transition est aussi une lutte fondamentalement politique entre acteurs et forces sociales pour déterminer le calibrage et la signification de cette transition. Le débat sur la place des ressources dans l'économie canadienne devrait donc être pensé comme un questionnement politique des ressources qui se situe simultanément à des échelles différentes, sous la forme d'une délibération ou d'une lutte en fonction des caractéristiques du territoire concernés, qu'ils soient urbains ou ruraux. Ce questionnement permettrait, en outre, d'envisager de nouvelle façon les rapports entre métropoles globales et territoires périphériques et ainsi réinvestir un questionnement fondateur dans les études régionales.

\section{BIBLIOGRAPHIE ET NOTES}

${ }^{1}$ Les auteurs sont professeurs à L'UQAR et membres du Centre de recherche sur le développement territorial (CRDT).

${ }^{2}$ Voir CÔTÉ, Serge et PROULX, Marc-Urbain (2002). L'économie des régions périphériques du Québec et son renouvellement actuel, Chicoutimi et Rimouski, Centre de recherche sur le développement territorial, $140 \mathrm{p}$.

${ }^{3}$ Cette étude a été réalisée avec nos collègues Geneviève Brisson (INSP), Guy Chiasson (UQO) et Marie-Claude Prémont (ENAP) dans le cadre de l'évaluation environnementale sur les gaz de schiste (Fournis, Fortin, 2013).

${ }^{4}$ Voir DRACHE, Daniel (1995). «Introduction : Celebrating Innis: The Man, the Legacy, and Our Future », in Harold A. Innis (dir.), Staples, Markets, and Cultural Change: Selected Essays, Centenary Ed., Montreal, McGill-Queen's University Press, p. xiii lix.
${ }^{5}$ Voir BARNES, Trevor (1996). « External shocks: regional implications of an open staple economy », in John N. H. Britton (dir.), Canada and the Global Economy: The Geography of Structural and Technological Change, Montreal, McGill-Queen's University Press, p. 48 68. Et WELLSTEAD, Adam (2007). «The (Post) Staples Economy and the (Post) Staples State in Historical Perspective », Canadian Political Science Review, vol. 1, ${ }^{\circ}$ 1, p. 825.

${ }^{6}$ Voir HOWLETT, Michael et BROWNSEY, Keith (2007). «Introduction to Special Issue on Canada's Staples Industries », Canadian Political Science Review, vol. 1, $\mathrm{n}^{\circ} 1$, p. 17.

${ }^{7}$ Selon le MRN, plus de $95,8 \%$ de $«$ la production totale d'électricité disponible au Québec » en 2010 (http://www.mrn.gouv.qc.ca/energie/statistiques/statis tiques-production-electricite.jsp, consultée le 25 février 2014).

${ }^{8}$ Voir SZARKA, Joseph (2007). Wind power in Europe : politics, business and society, Houndmills, Basingstoke, Hampshire; New York, N.Y, Palgrave Macmillan, coll. « Energy, climate, and the environment series », $228 \mathrm{p}$.

${ }^{9}$ Voir PASQUALETTI, Martin J. (2011). « The Next Generation of Energy Landscapes », in Stanley D. Brunn (dir.), Engineering Earth, Springer Netherlands, p. 461-482.

${ }^{10}$ Selon SOVACOOL et COOPER (2013), un mégaprojet énergétique serait un projet à grande échelle financière et géographique, qui mobiliserait des investissements d'un milliard de dollars sur une échelle transnationale (Sovacool, Benjamin $\mathrm{K}$ et Cooper, Christopher J (2013). The governance of energy megaprojects : politics, hubris and energy security, Cheltenham, UK, Edward Elgar, 272 p.)

${ }^{11} \mathrm{http}: / /$ www.edf-en.ca/press-display/post/65/EDFEN-Canada-et-Enbridge-inaugurent-le-parc-olien. ${ }^{12}$ Selon les données de l'étude SECOR-KPMG (2013) citées à la page 6 du mémoire soumis par le TechnoCentre Éolien lors de la Commission sur les enjeux énergétiques tenue en 2013.

${ }^{13}$ Voir HESSING, Melody et HOWLETT, Michael (1997). Canadian natural resource and environmental policy: political economy and public policy, Vancouver, UBC Press, 338 p. 